Original Article

\title{
The effectiveness of extracorporeal shockwave therapy for frozen shoulder in patients with diabetes: randomized control trial
}

\author{
Ramprasad Muthukrishnan, MSPT, PhD ${ }^{1}{ }^{*}$, Ayesha Abdul Rashid, BPT ${ }^{1)}$, \\ FATMA Al-AlkharJi, BPT ${ }^{2)}$ \\ 1) Physiotherapy Division, College of Health Sciences, Gulf Medical University: Ajman, Ajman, UAE \\ 2) Department of Physiotherapy, Umm Al Quwain Hospital, UAE
}

\begin{abstract}
Purpose] This study examined the effectiveness of extracorporeal shock wave therapy versus ultrasound therapy, combined with the mobilization and therapeutic exercise in both groups, in participants with diabetic frozen shoulder. [Participants and Methods] Twenty participants with diabetic frozen shoulder were divided into an experimental group who received extracorporeal shock wave therapy, mobilization and exercises $(\mathrm{n}=10$, Mean: 43.70) and the control group who received ultrasound, mobilization and exercises ( $\mathrm{n}=10$ Mean: 45.50). The clinical outcomes, i.e., a) pain b) active range of motions of the shoulder, c) disability scores by Disabilities of the Arm, Shoulder and Hand scale and d) global rating of change was measured weekly for four weeks. [Results] Significant improvements in pain, all active range of motions and disability scores were observed at the end of the 4th week in both groups. Additionally, the experimental group benefitted significant pain reduction (median difference: 7 in experimental versus 6 in control group), reduced number of therapy sessions and thus the costs of treatment compared to the control group. [Conclusion] Extracorporeal shock wave therapy significantly reduced pain in people with diabetic frozen shoulder with a reduction of treatment cost compared to the control group.

Key words: Extracorporeal shock wave therapy, Frozen shoulder, Diabetes
\end{abstract}

(This article was submitted Oct. 10, 2018, and was accepted Feb. 1, 2019)

\section{INTRODUCTION}

Frozen shoulder is a painful disorder with high to low irritability leading to stiffness and disability ${ }^{1)}$. The incidence of diabetic patients having frozen shoulder is five times higher and prevalence rate of $2-13 \%$ of frozen shoulder in diabetic patients $^{2)}$.

Literature elucidated that pain, functional disability and active range of motion for frozen shoulder decreased more markedly in participants with diabetes as compared to those without diabetes ${ }^{3)}$. On the other hand, pain scores remain elusive and no correlation between pain scores and diabetes was also reported ${ }^{4)}$. According to a synthesis of systematic reviews that revealed therapeutic exercises, mobilization helped to achieve short-term goals such as reducing pain and increasing active range of motion (AROM) as well as long-term goals that increased in activities of daily living and reduced the dependence on others for their work while compared to sham ultrasound in treating non-diabetic frozen shoulder ${ }^{5-7)}$. Further, it was reported that low level laser therapy was also beneficial ${ }^{5}$.

Ultrasound therapy provided significant beneficial effect only on pain reduction or acts as a placebo and served as an effective adjunct for therapeutic exercises ${ }^{8}$. Extracorporeal shock wave therapy (ESWT) has emerged as the treatment of choice for rotator cuff calcific tendinopathies for its efficacy in pain reduction, earlier return to daily activities and improved quality of life ${ }^{9,10)}$. It was stated that need for more research to find the effectiveness of ESWT in frozen shoulder, non-

*Corresponding author. Ramprasad Muthukrishnan (E-mail: mrp@gmu.ac.ae)

(C2019 The Society of Physical Therapy Science. Published by IPEC Inc.

(c) (1) $\Theta$ This is an open-access article distributed under the terms of the Creative Commons Attribution Non-Commercial No Derivatives cc) 
calcific tendinopathy ${ }^{10,11)}$ and the further need to find the effectiveness of diabetic frozen shoulder.

There remains a large gap in knowledge a) Does ESWT provide similar clinical improvement for diabetic frozen shoulder b) Can ESWT provide continuum of treatment for those refusing invasive procedures on diabetic frozen shoulder c) what are the effects of ESWT versus conservatively applied ultrasound combined with therapeutic exercise on diabetic frozen shoulder d) Is ESWT helpful in altering the course of disease progression on unresponsive diabetic frozen shoulders.

Till date only one has study reported the efficacy and clinical improvements obtained by ESWT on diabetic frozen shoulder ${ }^{10)}$. However, no studies have reported and compared other conventional physical therapy options. Keeping all views, the primary objective of the study was to compare the effectiveness of "ESWT with joint mobilization and exercises" with "ultrasound therapy with joint mobilization and exercises" on improving pain and functional recovery in participants with frozen diabetic shoulder. It was hypothesized that there will be a significant difference between extracorporeal shockwave therapy with joint mobilization and exercises and ultrasound therapy with joint mobilization and exercises in improving shoulder pain and functions in diabetic frozen shoulder.

\section{PARTICIPANTS AND METHODS}

Twenty participants with phase 1 and 2 Frozen shoulders ${ }^{12)}$ with diabetes were prospectively and randomly selected for the study based on the diagnosis of MRI findings, referred and/or registered for physiotherapy services. Using age matched stratification, participants were allocated into an experimental group ( $\mathrm{n}=10, \mathrm{M}: 4, \mathrm{~F}: 6$ with mean age $43.70 \pm 10.4)$ who were treated with ESWT, mobilization and therapeutic exercises and control group (n=10, M:3, F:7) with mean age 45.50 \pm 14.3 ) underwent ultrasound with mobilization and therapeutic exercises. The study was conducted at out-patient units of Umm-Al-Quwain hospital, Umm-Al-Quwain and Thumbay Hospital, Ajman, United Arab Emirates from February 2018 to September 2018.

The inclusion criteria were; a) relapsed or episode of pain score $\geq 5$ at assessment with a past history of pain for at least 2 months b) phase 1 or 2 frozen shoulder confirmed by a physician c) ROM restriction ( $>75 \%$ ROM loss in $\geq 2$ directions including abduction, flexion and external rotation) d) no treatment other than analgesics with unresponsive ROM restriction for the past 2 months e) unwilling or waiting and did not undergo joint injection and f) medical diagnosis of type 2 diabetes. The exclusion criteria were; a) patient with shoulder complaint and pain history of more than 12 months b) autoimmune mediated arthritis c) full thickness tear of rotator cuff d) neck pain with radiculopathy and e) abrasion on the affected shoulder.

The study was approved by the ethics committee and participants were informed of their right to withdraw anytime and any compensations for participation. An information sheet about ESWT, ultrasound therapy and therapeutic exercises and mobilization were explained, and all participants provided written informed consent. Prior to the treatment, all participants were physically examined ${ }^{13,14)}$ to determine the target tissue for the accurate delivery of the shock wave energy as well as ultrasound therapy. The control group was treated with a range of conservative physical therapy, including hot packs (10-12 minutes), ultrasound (6-8 minutes), mobilization and shoulder exercise which was based on the standard protocol for frozen shoulder ${ }^{15-20)}$. The experimental group received joint mobilization and exercises physical therapy, and then instead of ultrasound therapy participants were treated with a magnetic ESWT unit (STORZ Medical Masterplus MP50). Up to 2,000 times, shock waves with frequency of $3 \mathrm{~Hz}$ were applied using a focus-type head, while the intensity of energy was adjusted according to participant's tolerance of pain. The control group participants were treated three times a week over a four-week period. The ESWT group received once session per week and all participants underwent a follow-up session on 12th the week. Ten participants in each group completed the trial and one participant from the control group withdrew citing personal circumstances before the intervention. A CONSORT flow diagram is shown in Fig. 1.

The pre and post intervention measures included were; a) a visual analogue scale (VAS) which provides pain score ranges from $0-10$ where 0 indicates no pain and 10 indicates extremely painful ${ }^{21)}$.

The Disabilities of the Arm, Shoulder and Hand (DASH) questionnaire was a 30-item questionnaire that measures the ability of a patient to perform certain upper extremity tasks. The scores obtained provide disability status and changes in symptoms and function over a period ${ }^{22}$.

GROC Score: This rating scale was used to rate the overall outcome of the condition after physical therapy intervention. The Global Rating of Change (GROC) was used to provide a comparison between outcome measures ${ }^{23}$. Non-parametric test 
Table 1. Baseline characteristics of the study participants

\begin{tabular}{lcc}
\hline \multicolumn{1}{c}{ Group } & Experimental & Control \\
\hline Age (years) & $43.70 \pm 10.4$ & $45.5 \pm 14.3$ \\
Height $(\mathrm{cm})$ & $161.4 \pm 5.97$ & $164.5 \pm 5.4$ \\
Weight $(\mathrm{kg})$ & $86.6 \pm 8.9$ & $89.1 \pm 8.5$ \\
Duration of disease* (in months) & $7.38 \pm 2.29$ & $7.97 \pm 2.80$ \\
Fasting blood glucose value (mg/dL) & $130.0 \pm 6.15$ & $131.3 \pm 6.42$ \\
\hline
\end{tabular}

Values are mean \pm standard deviation.

*Frozen shoulder.

Table 2. Pre and post treatment analysis of pain (VAS), disability (DASH), and change (GROC) and shoulder active range of motions within the ESWT group

\begin{tabular}{lccccccc}
\hline \multirow{2}{*}{ Variable } & \multicolumn{3}{c}{ Rank } & & \multicolumn{3}{c}{ Median } \\
\cline { 2 - 3 } \cline { 6 - 7 } VAS & Negative & Positive & & Pre & Post & Difference \\
\hline DASH & 10 & 0 & & 9 & 1.5 & $-7 *$ \\
GROC & 10 & 0 & & 39.4 & 19.9 & $-19.2^{*}$ \\
AROM-ABD & 0 & 10 & & -3.5 & 5.5 & $9 *$ \\
AROM-FLEX & 0 & 10 & & 95 & 142.5 & $43.5^{*}$ \\
AROM-ER & 0 & 10 & & 96 & 140.5 & $46^{*}$ \\
\hline
\end{tabular}

*Significant $\mathrm{p}$ value $(<0.05)$.

VAS: Visual analog scale for pain; DASH: Disabilities of the arm, shoulder and hand; GROC: Global rating of change; AROM: Active range of motion in degrees; ABD: Shoulder Abduction; FLEX: Shoulder flexion; ER: Shoulder external rotation.

Table 3. Pre and post treatment analysis of pain (VAS), disability (DASH), and change (GROC) and shoulder active range of motion within the control group

\begin{tabular}{lcccccc}
\hline \multirow{2}{*}{ Variable } & \multicolumn{3}{c}{ Rank } & & \multicolumn{3}{c}{ Median } \\
\cline { 2 - 3 } \cline { 5 - 6 } Vegative & Positive & & Pre & Post & Difference \\
\hline VAS & 10 & 0 & & 9 & 3.5 & $-6^{*}$ \\
DASH & 10 & 0 & & 43.4 & 29.15 & $-14.1^{*}$ \\
GROC & 0 & 10 & & -3 & 5 & $8^{*}$ \\
AROM-ABD & 0 & 10 & & 90 & 142 & $48^{*}$ \\
AROM-FLEX & 0 & 10 & & 105 & 142 & $41^{*}$ \\
AROM-ER & 0 & 10 & & 42.5 & 65 & $23.5^{*}$ \\
\hline
\end{tabular}

*Significant $\mathrm{p}$ value $(<0.05)$.

VAS: Visual analog scale for pain; DASH: Disabilities of the arm, shoulder and hand; GROC: Global rating of change; AROM: Active range of motion in degrees; ABD: Shoulder Abduction; FLEX: Shoulder flexion; ER: Shoulder external rotation.

statistic tests were performed; Mann-Whitney U test and Wilcoxon signed rank test to compare the variables in pre and post intervention. A p-value of less than 0.05 was considered statistically significant.

\section{RESULTS}

The participants' baseline characteristics were reported in Table 1. Within group pre and post comparison after 4 weeks of ESWT and control group revealed significant improvement in median difference of pain ( $-7 \mathrm{vs} .-6)$, DASH (-19.2 vs. -14.1$)$, GROC scores (9 vs. 8), AROM shoulder flexion $\left(46^{\circ}\right.$ vs. $\left.41^{\circ}\right)$, AROM shoulder external rotation $\left(28^{\circ}\right.$ vs. $\left.23.5^{\circ}\right)$ and $\mathrm{AROM}$ shoulder abduction $\left(43.5^{\circ}\right.$ vs. $\left.48^{\circ}\right),(\mathrm{p}<0.05$, Table 2 and Table 3$)$. Progressions throughout the 4 weeks were reported in Table 4 and Table 5, for the ESWT and Control group, respectively. Intergroup comparison of post intervention difference was analyzed using Mann-Whitney U test which revealed significant improvement only in pain scores favoring ESWT $(\mathrm{p}<0.05)$ at the end of 4 th week.

\section{DISCUSSION}

This trial was pragmatic in nature, conducted in physiotherapy outpatient departments, where participants registered physiotherapy for services phase 1 and 2 frozen shoulder with type 2 diabetes. The participant had 5 to 10 months of untreated, 
Table 4. Weekly comparison of progression of clinical outcomes for ESWT group

\begin{tabular}{lcccc}
\hline ESWT group $(\mathrm{n}=10)$ & Week 1 & Week 2 & Week 3 & Week 4 \\
\hline VAS & $8.60 \pm 0.84$ & $6.40 \pm 0.843$ & $3.70 \pm 1.49$ & $1.60 \pm 0.7$ \\
DASH & $38.7 \pm 8.3$ & $33.4 \pm 7.0$ & $26.5 \pm 5.82$ & $20.2 \pm 5.73$ \\
GROC & $3.80 \pm 1.4$ & $0.50 \pm 1.9$ & $3.30 \pm 1.89$ & $5.40 \pm 1.71$ \\
AROM-ABD & $95.40 \pm 20.1$ & $117 \pm 13.5$ & $130 \pm 11.7$ & $142 \pm 11.5$ \\
AROM-FLEX & $94.40 \pm 30.5$ & $118 \pm 17.5$ & $133.40 \pm 15.2$ & $146 \pm 16.8$ \\
AROM-ER & $52.80 \pm 18.3$ & $59.80 \pm 16.6$ & $71.60 \pm 14.7$ & $76.90 \pm 10.7$ \\
\hline
\end{tabular}

Values are mean \pm standard deviation.

VAS: Visual analog scale for pain; DASH: Disabilities of the arm, shoulder and hand; GROC: Global rating of change; AROM: Active range of motion in degrees; ABD: Shoulder Abduction; FLEX: Shoulder flexion; ER: Shoulder external rotation.

Table 5. Weekly comparison of progression of clinical outcomes for control group

\begin{tabular}{lcccc}
\hline Control group $(\mathrm{n}=10)$ & Week 1 & Week 2 & Week 3 & Week 4 \\
\hline VAS & $9.40 \pm 0.516$ & $7.40 \pm 0.516$ & $5.40 \pm 0.7$ & $3.30 \pm 0.82$ \\
DASH & $45 \pm 7.96$ & $40.130 \pm 7.44$ & $35.4 \pm 5.51$ & $29.9 \pm 5.14$ \\
GROC & $-2.80 \pm 1.03$ & $0.10 \pm 1.45$ & $2.80 \pm 1.03$ & $5.10 \pm 0.738$ \\
AROM-ABD & $101 \pm 19.1$ & $115 \pm 18$ & $133 \pm 15.2$ & $149 \pm 15.7$ \\
AROM-FLEX & $97.50 \pm 19.9$ & $118 \pm 13.8$ & $129 \pm 10.5$ & $141 \pm 5.76$ \\
AROM-ER & $41.00 \pm 5.68$ & $51.7 \pm 6.31$ & $58.0 \pm 5.4$ & $64.6 \pm 3.47$ \\
\hline
\end{tabular}

Values are mean \pm standard deviation.

VAS: Visual analog scale for pain; DASH: Disabilities of the arm, shoulder and hand; GROC: Global rating of change; AROM: Active range of motion in degrees; ABD: Shoulder Abduction; FLEX: Shoulder flexion; ER: Shoulder external rotation.

recalcitrant frozen shoulder with a recurrence of higher pain scores (up to 8) with 75\% loss in the range of motion in two directions and unwilling to undergo or waiting for invasive procedures. This trial elucidated the effectiveness of ESWT on clinical improvement in short term (4 sessions) with high to moderate irritable frozen shoulder. In this study, ESWT is effective, feasible, and well tolerated and served as a valid alternative to glenohumeral joint injections for participants with phase 1 and 2 frozen shoulder with diabetes without any side-effects. This study results were corroborated with other preliminary studies on diabetic frozen shoulder and without diabetes $\left.{ }^{7}, 10\right)$.

The ESWT group achieved significant improvements with 4 ESWT and augmented therapeutic exercise sessions per month whereas the control group received 12 sessions per month to achieve similar results (Table 2 and 3 ). Intergroup comparison on post intervention of clinical outcomes using A Mann-Whitney $U$ test revealed that statistically significant median scores for reduction in pain benefitting ESWT group (median pain score: -7$)$ and not for other clinical outcomes such as disability, active range of motions of shoulder, and satisfaction scores $(\mathrm{U}=20.5, \mathrm{z}=-2.42, \mathrm{p}=0.023)$.

An increase in the active range of motion in abduction was higher in the control group than ESWT group by up to 5 degrees. This may be due to the difference in the local anterolateral application of ultrasound to different points of the shoulder capsule and higher therapeutic exercise sessions in the control group compared to the ESWT group.

In this study, all participants were provided with a follow up session at the end of 12th week. Follow-up of clinical outcomes at end of the 12th week revealed no significant difference between control and ESWT group. It was evident that the ESWT group demonstrated early improvements in pain reduction within four weeks as compared to the control group, which achieved similar pain reduction at 12th week. This could be attributed to additional eight sessions of prescribed treatment received by ultrasound with therapeutic exercises group or resolution of frozen shoulder. A simple cost effectiveness analysis was performed. It revealed that ESWT combined physiotherapy sessions were 3 times less expensive than the control group.

One of the limitations of the study was the small sample, constrained with economic and logistical reasons; hence results could be viewed cautiously. However, this study provided valuable inputs on the early progression of clinical outcomes achieved by ESWT. Participants were empowered to take necessary medical therapy for the control of blood glucose level throughout the study period. Secondly, due to high pain scores, restriction in range of motion and discomfort, participants were positioned in their comfort zone of the shoulder during the ESWT and ultrasound application. However, it was reported that ESWT applied in hyper-extended position (66.6\% absorption) had a better recovery in range of motion compared to the neutral position $(35.3 \% \text { absorption })^{24)}$. The age range of participants was from 31-60 years, and a larger sample, stratification based on disease phases, irritability levels and age groups would have provided better insights on which age group and phases benefitted more by ESWT or ultrasound, both augmented with therapeutic exercises. The empowered self-monitoring of blood glucose control during the trial and the effects of an additional session of exercises on the improvement of clinical outcomes could not be differentiated. 


\section{Funding and Conflict of interest}

This study was supported by a research grant from Gulf Medical University.

\section{ACKNOWLEDGEMENTS}

We thank Dr. Praveen Kumar, Mr. Satheesh Kumar, Ms. Annamma Matthew, Ms. Sivapriya, Dr. Prathap, Dr. Kumar Guru, Dr. Jagatheesan, Faculty and clinical physiotherapists of Thumbay Hospital, Ajman and Physiotherapy Department, Umm Al Quwain Hospital for their support during protocol-based treatment and invaluable inputs throughout the study. We thank Dr. Anusha Sreejith for help formulating the RCT and statistical analysis.

\section{REFERENCES}

1) Kelley MJ, McClure PW, Leggin BG: Frozen shoulder: evidence and a proposed model guiding rehabilitation. J Orthop Sports Phys Ther, 2009, 39: 135-148. [Medline] [CrossRef]

2) Zreik NH, Malik RA, Charalambous CP: Adhesive capsulitis of the shoulder and diabetes: a meta-analysis of prevalence. Muscles Ligaments Tendons J, 2016, 6: 26-34. [Medline] [CrossRef]

3) Smith LL, Burnet SP, McNeil JD: Musculoskeletal manifestations of diabetes mellitus. Br J Sports Med, 2003, 37: 30-35. [Medline] [CrossRef]

4) Jain TK, Sharma NK: The effectiveness of physiotherapeutic interventions in treatment of frozen shoulder/adhesive capsulitis: a systematic review. J Back Musculoskeletal Rehabil, 2014, 27: 247-273. [Medline] [CrossRef]

5) Page MJ, Green S, Kramer S, et al.: Electrotherapy modalities for adhesive capsulitis (frozen shoulder). Cochrane Database Syst Rev, 2014, (10): CD011324. [Medline]

6) Ebadi S, Forogh B, Fallah E, et al.: Does ultrasound therapy add to the effects of exercise and mobilization in frozen shoulder? A pilot randomized double-blind clinical trial. J Bodyw Mov Ther, 2017, 21: 781-787. [Medline] [CrossRef]

7) Kyhlbäck M, Schröder Winter H, Thierfelder T, et al.: Physiotherapy treatment of the diabetic shoulder: a longitudinal study following patients with diabetes and shoulder pain using a pre-post treatment design. Disabil Rehabil, 2014, 36: 556-562. [Medline] [CrossRef]

8) Chen CY, Hu CC, Weng PW, et al.: Extracorporeal shockwave therapy improves short-term functional outcomes of shoulder adhesive capsulitis. J Shoulder Elbow Surg, 2014, 23: 1843-1851. [Medline] [CrossRef]

9) Moya D, Ramón S, Guiloff L, et al.: Current knowledge on evidence-based shockwave treatments for shoulder pathology. Int J Surg, 2015, 24: 171-178. [Medline] [CrossRef]

10) Santoboni F, Balducci S, D’Errico V, et al.: Extracorporeal shockwave therapy improves functional outcomes of adhesive capsulitis of the shoulder in patients with diabetes. Diabetes Care, 2017, 40: e12-e13. [Medline] [CrossRef]

11) Vahdatpour B, Taheri P, Zade AZ, et al.: Efficacy of extracorporeal shockwave therapy in frozen shoulder. Int J Prev Med, 2014, 5: 875-881. [Medline]

12) Rangan A, Goodchild L, Gibson J, et al.: Frozen shoulder. Shoulder Elbow, 2015, 7: 299-307. [Medline] [CrossRef]

13) Occupational Therapy Assessment Guide: https://otassessments.wordpress.com/events-list/shoulder/ (Accessed Oct. 6, 2018)

14) Stacommunications.com: http://www.stacommunications.com/journals/cme/images/cmepdf/dec01/shoulder.pdf. (Accessed Oct. 9, 2018)

15) Bal A, Eksioglu E, Gulec B, et al.: Effectiveness of corticosteroid injection in adhesive capsulitis. Clin Rehabil, 2008, 22: 503-512. [Medline] [CrossRef]

16) Dias R, Cutts S, Massoud S: Frozen shoulder. BMJ, 2005, 331: 1453-1456. [Medline] [CrossRef]

17) Gaspar PD, Willis FB: Adhesive capsulitis and dynamic splinting: a controlled, cohort study. BMC Musculoskelet Disord, 2009, 10: 111. [Medline] [CrossRef]

18) Kelley M, Mcclure P, Leggin B: Frozen sholder: envidence and a proposed model guiding rehabilitation. J Orthop Sports Phys Ther, 2009, 39: 135-148. [Medline] [CrossRef]

19) Ruiz JO: Positional stretching of the coracohumeral ligament on a patient with adhesive capsulitis: a case report. J Manual Manip Ther, 2009, 17: 58-63. [Medline] [CrossRef]

20) Vermeulen HM, Rozing PM, Obermann WR, et al.: Comparison of high-grade and low-grade mobilization techniques in the management of adhesive capsulitis of the shoulder: randomized controlled trial. Phys Ther, 2006, 86: 355-368. [Medline]

21) Physiopedia: Visual analogue scale. https://www.physio-pedia.com/Visual_Analogue_Scale (Accessed Oct. 10, 2018)

22) Orthopedic Score: The Disabilities of the Arm, Shoulder and Hand (DASH) Score. http://orthopaedicscore.com/scorepages/disabilities_of_arm_shoulder_ hand_score_dash.html (Accessed Oct. 10, 2018)

23) Cisco: Global rating of change scale (GROC). https://www.cisco.com/web/lchc/assets/pdf/GROC.pdf (Accessed Oct. 10, 2018)

24) Tornese D, Mattei E, Bandi M, et al.: Arm position during extracorporeal shock wave therapy for calcifying tendinitis of the shoulder: a randomized study. Clin Rehabil, 2011, 25: 731-739. [Medline] [CrossRef] 